\title{
Perfil das solicitações de medicamentos de alto custo ao Sistema Único de Saúde em Minas Gerais
}

\author{
Rilke Novato Públio \\ Secretaria Municipal de Saúde de Betim (MG) \\ Braulio Roberto Gonçalves Marinho Couto \\ Centro Universitário Belo Horizonte (UNI-BH) \\ Analina Furtado Valadão \\ Instituto Metropolitano do Ensino Superior (Imes/Univaço) \\ Edna Maria Rezende \\ Escola de Enfermagem (UFMG)
}

\begin{abstract}
O acesso universal aos serviços de saúde, previsto na Constituição Federal, deveria garantir assistência terapêutica integral, inclusive de medicamentos do componente especializado (de alto custo). Considerando que esse é um dos maiores desafios ao Sistema Único de Saúde, o objetivo deste estudo foi analisar custos do fornecimento desses medicamentos aos usuários de planos privados de saúde, pela Secretaria Estadual de Saúde, no estado de Minas Gerais. Foram selecionados processos de solicitações de medicamentos do componente especializado deferidos pela Secretaria Estadual de Saúde, no primeiro semestre de 2008. O método record linkage foi utilizado para verificar junto à Agência Nacional de Saúde Suplementar, se os solicitantes eram ou não beneficiários de planos privados de saúde. Os dados mostraram que cerca de $37 \%$ dos gastos com esses medicamentos foram de solicitações dos usuários de planos privados e que o custo mediano desses medicamentos foi mais elevado, com diferença significativa, em relação às solicitações dos usuários que não tinham plano. Observou-se ainda que o custo dos medicamentos solicitados pela Gerência Regional de Saúde de Belo Horizonte foi significativamente mais alto em relação às demais. Conclui-se pela necessidade de aprofundar estudos sobre implementação de políticas de assistência farmacêutica pelos planos privados de saúde, no sentido de minimizar os gastos públicos.
\end{abstract}

Palavras-chave: medicamentos do componente especializado; planos de saúde; gastos em saúde; sistema único de saúde.

DOI: http://dx.doi.org/10.1590/0034-76121513

Artigo recebido em 23 mar. 2013 e aceito em 26 jun. 2014.

Rev. Adm. Pública - Rio de Janeiro 48(6):1567-1585, nov./dez. 2014 


\section{Perfil de solicitudes de medicamentos de alto costo en el Sistema Único de Salud en Minas Gerais}

El acceso universal a los servicios de salud por parte de la Constitución Federal, debe garantizar la atención integral, incluyendo componente especializado de los medicamentos (alto costo). Teniendo en cuenta que este es uno de los mayores retos para el Sistema de Salud, el objetivo de este estudio fue analizar los costos de la prestación de estos medicamentos para los usuarios de los planes privados de salud, el Departamento de Salud del estado, estado de Minas Gerais. Método de vinculación de registros se seleccionaron Procesa las solicitudes de componente especializado de los medicamentos rechazados por el Departamento de Salud del Estado, en el primer semestre de 2008. Se utilizó para comprobar con la Agencia Nacional de Salud, si los demandantes eran beneficiarios de los planes privados la salud. Los datos mostraron que cerca del $37 \%$ del gasto en estos medicamentos eran las peticiones de los usuarios de los planes privados y la mediana del costo de estos medicamentos fue mayor, con una diferencia significativa en relación a las solicitudes de los usuarios que no tenían ningún plan. También se observó que el costo de los medicamentos solicitados por la Administración de Salud Regional de Belo Horizonte fue significativamente mayor en relación con el otro. Se concluye la necesidad de realizar más estudios sobre la aplicación de políticas de atención farmacéutica por planes de salud privados con el fin de minimizar el gasto público.

Palabras clave: drogas de componente especializado; los planes de salud; los gastos en salud; sistema único de salud.

Profile of requests for high cost drugs to the Unified Health System in Minas Gerais, Brazil Universal access to health services, predicted by the Federal Constitution, should ensure integrated care, including specialized component medications (high cost). Considering that this is one of the biggest challenges to the Unified Health System, the aim of this study was to analyze the costs of providing these drugs to users of private health plans, by the state Department of Health of Minas Gerais, Brazil. Processes requests for specialized component of the medications rejected by the State Department of Health in the first semester of 2008 were selected. Record Linkage's method was used to check with the National Health Agency, whether or not the applicants were beneficiaries of health private plans. The data showed that about $37 \%$ of the expenses on these drugs were requests from users of private plans and the median cost of these medications was higher, with a significant difference in relation to requests from users who had no plan. It was also observed that the cost of drugs requested by the Regional Health Management of Belo Horizonte was significantly higher when compared to others. We can conclude that it is necessary further studies on policy implementation of pharmaceutical care by private health plans in order to minimize public expenses.

Keywords: drugs of specialized component; health plans; health expenses; unified health system.

\section{Introdução}

O acesso a medicamentos no Brasil sempre foi identificado como um dos maiores desafios da saúde. A assistência farmacêutica pode ser considerada uma das ações que integram os serviços de saúde, embora, historicamente, tenha sido tratada como um serviço à parte (Aquino, 2008). 
O acesso universal e integral aos serviços de saúde está previsto na Constituição Federal do país. Esse direito constitucional foi regulamentado pela Lei no 8.080/1990 que, dentre outras ações, estabeleceu que o SUS deveria ser estruturado de forma a garantir assistência terapêutica integral, inclusive assistência farmacêutica (Brasil, 2000).

O Brasil, segundo estimativas da Fundação Instituto Brasileiro de Geografia e Estatística (FIBGE), tem um dispêndio total em saúde (público e privado) equivalente a 8,4\% do PIB, percentual baixo quando comparado aos Estados Unidos (15,47\%), que possui política de saúde alicerçada no sistema privado. O gasto público investido em saúde no Brasil representa apenas $41,6 \%$ do gasto total, muito pouco para o país ter, efetivamente, um sistema de cobertura universal e atendimento integral. Entre os países da Organização para Cooperação do Desenvolvimento Econômico (Ocde), a participação das fontes públicas no financiamento do sistema representa em média 70\% da despesa total, variando de 67,5\% na Austrália a 84,1\% na Noruega (Fibge, 2010).

No que se refere ao acesso aos medicamentos no país, segundo dados divulgados pela Fibge, as despesas pelo desembolso financeiro direto com medicamentos responderam por $48,6 \%$ dos $\mathrm{R} \$ 153,81$ gastos mensalmente, em média, pelas famílias brasileiras. Em relação à saúde suplementar, registra-se que a procura por serviços de saúde ofertados pelos planos privados é cada vez maior (Oliveira et al., 2007). O número de beneficiários de planos de saúde no Brasil atingiu 48,66 milhões em setembro de 2012 (Brasil, 2013a).

A Lei no 9.656 , publicada em 1998, regulamenta os planos privados de saúde no Brasil e estabelece regras ao funcionamento da saúde suplementar, em face do caráter de complementaridade previsto na Constituição Federal de 1988 à iniciativa privada. Essa lei obriga aos planos privados de saúde a cobertura do fornecimento de medicamentos somente aos seus beneficiários que estiverem em regime de internação hospitalar, isentando os planos da obrigatoriedade de cobertura de medicamentos de uso domiciliar.

A decisão de instituir o fornecimento dos medicamentos do componente especializado foi estabelecida a partir da Portaria Interministerial n 3, de 15 de dezembro de 1982 . O propósito dessa Portaria era possibilitar o fornecimento pelo setor público de saúde, em caráter de excepcionalidade, de medicamentos a serem utilizados em algumas doenças de maior gravidade à saúde dos pacientes e que não constavam da Relação Nacional de Medicamentos (Rename). Para a provisão desses medicamentos eram exigidas a devida justificativa do prescritor e a autorização do gestor de saúde. O conceito de medicamento do componente especializado, antigamente denominado como excepcional, ou de alto custo, ou ainda de dispensação em caráter excepcional, foi se consolidando como aquele utilizado em doenças raras, atendendo a casos específicos. Geralmente é utilizado no tratamento de doenças crônicas, consideradas de caráter individual e que, a despeito de atingirem um número reduzido de pessoas, requerem tratamento longo ou até mesmo permanente. Um exemplo comum é o medicamento imunossupressor azatioprina $50 \mathrm{mg}$ a ser utilizado por pacientes que se submeteram ao transplante renal. Esse medicamento não integra as listas padronizadas de medicamentos nos municípios e sim da relação dos medicamentos do componente especiali- 
zado, sendo solicitado seu fornecimento às secretarias estaduais de saúde, em cada uma das unidades federativas.

O objetivo do presente estudo foi analisar os custos financeiros decorrentes do fornecimento de medicamentos do componente especializado aos beneficiários de planos privados de saúde pela Secretaria Estadual de Saúde de Minas Gerais (SES-MG). Essa situação é cada vez mais recorrente e vem preocupando autoridades públicas, gestores das operadoras de planos de saúde e, sobretudo, os usuários, necessitando, assim, de maiores estudos para o processo de gestão.

\section{Referencial teórico}

\subsection{0 desafio do acesso a medicamentos e o Sistema Único de Saúde}

A assistência farmacêutica no Brasil passou a fazer parte do rol de deveres do Estado, prevista no capítulo da saúde da Constituição Federal de 1988 e referendada pela Lei no 8.080, de 19 de setembro de 1990, conhecida como "Lei Orgânica de Saúde", que dispõe sobre os princípios fundamentais que regulamentam o SUS (Brasil, 2000).

O setor saúde no Brasil é marcado pela crescente demanda de medicamentos, em parte atribuída ao envelhecimento populacional e à mudança do perfil epidemiológico, com predomínio das doenças crônico-degenerativas em substituição às infectoparasitárias. Ainda, a propaganda abusiva de medicamentos estimula o consumo e propaga os lançamentos da indústria, propiciando uma imagem distorcida das necessidades sanitárias e criando um ideal de saúde inalcançável (Martins, 2004; Rodrigues, 2003; Soares, 2008). Segundo Lefréve e Lefréve (2004), existe uma inter-relação direta entre o sistema produtivo de serviços e produtos de saúde que insufla a busca por "mais saúde" e invariavelmente impõe interesses mercadológicos acima dos interesses técnicos necessários ao bem-estar do indivíduo.

Paralelamente, observa-se uma desenfreada incorporação tecnológica sem a devida regulação e avaliação da qualidade dos produtos que são colocados no mercado de saúde. Sabe-se que, apesar do alto custo justificado pela indústria para cobrir os gastos com pesquisa e desenvolvimento, a maioria desses produtos não representa inovações terapêuticas e são indicados para atender às demandas de países desenvolvidos ou para doenças "criadas" pela própria indústria farmacêutica, a exemplo da denominada "distonia do fuso horário", para a qual se pesquisa um novo fármaco para que os executivos que fazem longas viagens entre países com grande diferença de fuso horário possam adaptar-se o mais rápido possível tomando esse novo medicamento. Sabe-se que, com algumas horas de repouso, essa adaptação naturalmente ocorre (Angell, 2008; Oliveira et al., 2007).

O desenvolvimento dos fármacos é guiado pela lógica do capitalismo e focado em produtos com alto retorno financeiro em detrimento das prioridades sanitárias da população (Oliveira et al., 2007; Vidotti et al., 2008). São comuns os medicamentos de "imitação" que 
se assemelham aos antigos em relação à eficácia e segurança, porém confundem prescritores, dispensadores e gestores no momento de selecionar os medicamentos para seus pacientes ou para incluí-los em programas de assistência farmacêutica. Nesse cenário, o SUS, com a atribuição de garantir a todos o direito à saúde, tornou-se um grande mercado consumidor para os novos lançamentos da indústria farmacêutica (Carvalho, 2006).

Numa tentativa de possibilitar maior acesso aos medicamentos pelo poder de compra da população diretamente no mercado em 1993, o governo federal sancionou o DecretoLei n⿳⺈ 793, com o propósito de introduzir e estimular no Brasil a fabricação de produtos genéricos pelos laboratórios farmacêuticos instalados no País (Brasil, 2007). Os genéricos são cópias idênticas dos medicamentos de referência e, portanto, apresentam mesma composição química, forma farmacêutica, biodisponibilidade e podem ser fabricados e comercializados por diversos fabricantes em função de não se encontrarem sob regime patentário. Logo, não sendo mais objeto de monopólio, a concorrência no mercado contribui para a queda de preços. A partir desse decreto, o governo esperava que, autorizando oficialmente a fabricação dos genéricos, os laboratórios nacionais poderiam ter maior capacidade de competição no mercado e os preços dos medicamentos tenderiam a cair, possibilitando mais acesso pela população.

Todavia, a publicação do decreto-lei não se traduziu na prática, visto que sofreu poderosa resistência por parte das indústrias farmacêuticas estrangeiras (Barros, 2004). A política de medicamentos genéricos, após muitos anos de discussão junto à sociedade civil e com forte ameaça de retaliações dos fabricantes estrangeiros, foi finalmente aprovada pelo Congresso Nacional em 10 de fevereiro de 1999, com a expectativa de resgatar o princípio da maior oferta de medicamentos concorrentes no mercado e a queda de preços, possibilitando, assim, mais acesso (Brasil, 2000).

Em 1996, com a aprovação da Lei sobre a Propriedade Industrial (Lei das Patentes) pelo Congresso Nacional, verificou-se aumento das dificuldades quanto ao acesso aos medicamentos. Naquele momento, interessava às indústrias farmacêuticas estrangeiras aumentarem a importação dos chamados novos medicamentos patenteados, o que provocou maior crescimento na balança de importações, gerando maior dependência externa. Consequentemente, os preços tiveram elevações consideráveis, dificultando ainda mais o acesso (Oliveira et al., 2007).

Com a publicação da Portaria no 3.916 , em 1998, conhecida como "Política Nacional de Medicamentos", foram definidas diretrizes importantes voltadas à organização estrutural para o acesso aos medicamentos, sobretudo no tocante à aquisição, distribuição e disponibilização aos usuários. Aprovada pelo Conselho Nacional de Saúde, instância máxima do controle social, e pela Comissão Intergestores Tripartite, instância constituída pela representação dos gestores de saúde nas três esferas de governo, faltou a essa portaria a participação da sociedade em sua discussão e elaboração. Outro aspecto de destaque é a definição das responsabilidades de cada uma das esferas de governo sobre as ações referentes ao setor de medicamentos (Nascimento, 2002). 
No ano de 2004, o Conselho Nacional de Saúde aprova a Política Nacional de Assistência Farmacêutica (Pnaf), por meio da Resolução no 330/04, estabelecendo-a como parte da Política Nacional de Saúde, envolvendo um conjunto de ações voltadas à promoção, proteção e recuperação da saúde, e garantindo os princípios da universalidade, integralidade e equidade. Essa resolução define a assistência farmacêutica como um conjunto de ações voltadas à saúde, tanto individual como coletiva, tendo o medicamento como insumo essencial e visando o acesso e o uso racional (Brasil, 2007).

Na execução das ações de assistência farmacêutica, recomenda-se que o eixo do trabalho não seja somente o medicamento. É preciso ampliar a percepção do sistema para além da gestão da cadeia do fármaco, assegurando a integralidade das ações que permeiam o campo da intersetorialidade e agrega competências multiprofissionais (Perini, 2003).

Com o objetivo de organizar e dar orientação às ações da assistência farmacêutica, a Pnaf estabeleceu os seguintes componentes: assistência farmacêutica na atenção primária, medicamentos estratégicos e medicamentos excepcionais. O componente da atenção básica estabelece os fármacos inseridos na atenção primária e em alguns programas específicos de saúde, a exemplo da hipertensão, diabetes, saúde mental, tabagismo, entre outros. Os medicamentos estratégicos são previstos para o tratamento de doenças que se caracterizam como problemas de saúde pública e que podem atingir ou pôr em risco as coletividades, tais como tuberculose, hanseníase, malária, entre outras. Os medicamentos excepcionais são aqueles adquiridos em caráter de excepcionalidade em função das especificidades das patologias e também do alto custo dos fármacos (Brasil, 2007).

\subsection{Gerência Regional de Saúde (GRS)}

A Secretaria Estadual de Saúde de Minas Gerais (SES/MG) estabeleceu em seu organograma a divisão político-administrativa contemplando 28 (vinte e oito) Gerências Regionais de Saúde (figura 1).

Essas Gerências possuem competências no campo administrativo e técnico, reproduzindo em sua jurisdição as diretrizes e políticas com objetivo da promoção da descentralização das políticas de saúde do Estado. As gerências foram instituídas em bases jurídicas, com reconhecimento legal por parte da Secretaria e lhes cabem atribuições importantes como a garantia da gestão do sistema estadual de saúde em sua área de abrangência, a implementação das políticas estaduais, o assessoramento e o apoio às secretarias municipais de saúde naquilo que lhes couber e que estiver em consonância com a política estadual de saúde. São dotadas de infraestrutura física e de recursos humanos para proverem à demanda regional. No tocante à demanda por medicamentos excepcionais, cabe às gerências regionais a recepção de toda a documentação exigida ao processo de análise e cadastro dos solicitantes a ser enviada ao nível central da SES/MG para dar encaminhamentos. Cabe ao nível central a análise documental e técnica para proceder ao deferimento ou não da solicitação (Brasil, 2009). 
Figura 1

Divisão do estado de Minas Gerais em Gerências Regionais de Saúde

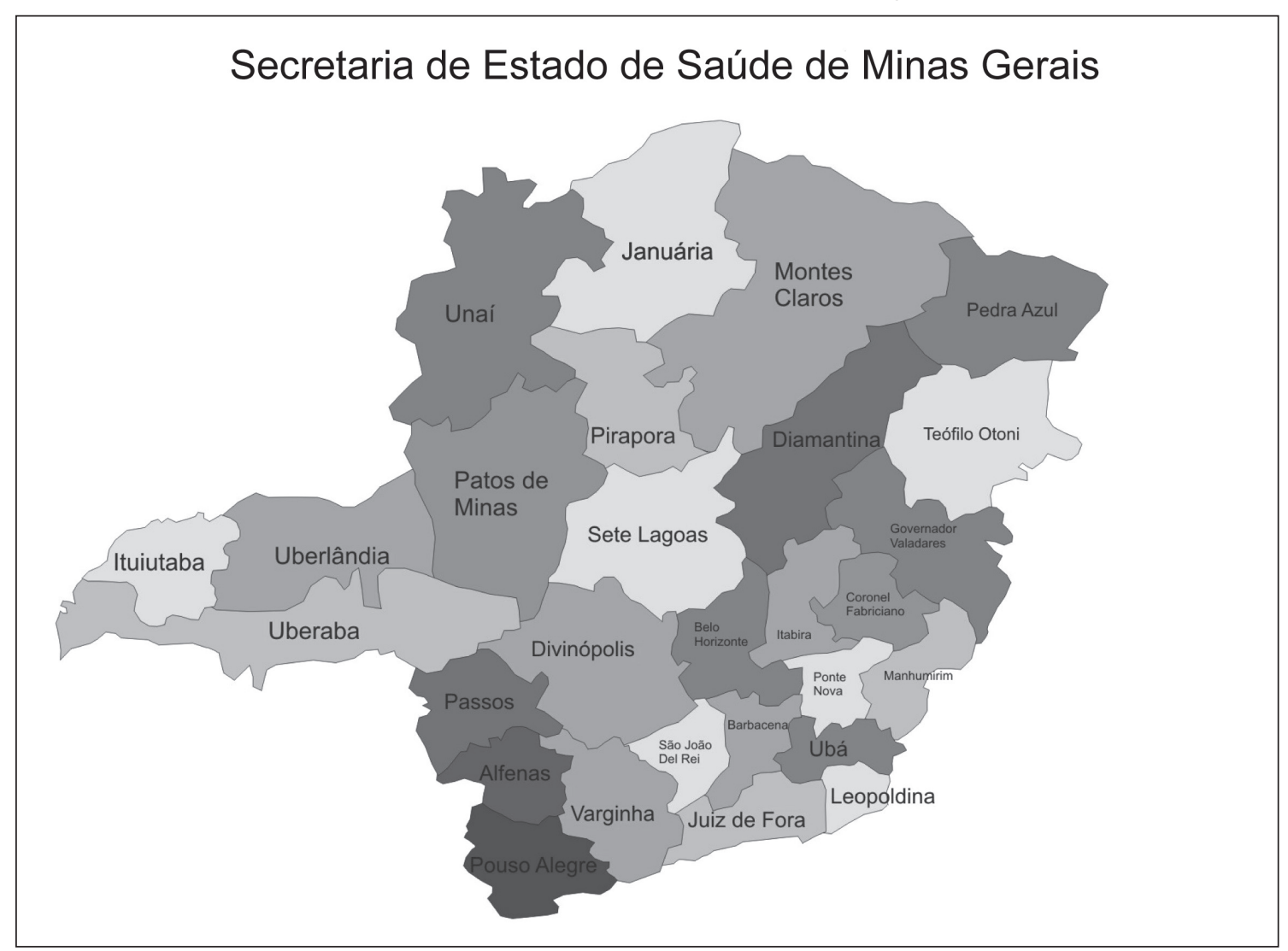

Fonte: Brasil (2011b).

\subsection{Saúde suplementar e assistência farmacêutica}

Na primeira metade do século XX inicia-se o processo de conformação e estruturação da saúde previdenciária no Brasil. Destaca-se o modelo do seguro social organizado em torno dos Institutos de Aposentadorias e Pensões (IAPs) que abrangiam diversas categorias de trabalhadores urbanos (Brasil, 2007). Para atendimento às demandas de saúde dos empregados associados, esses institutos, em sua grande maioria, compravam serviços de consultórios médicos ou de estabelecimentos hospitalares (Montone, 2004).

Na década de 1950, com o surgimento de grandes empresas estatais, a exemplo da Petrobras e o estabelecimento de empresas estrangeiras no país, aparecem os sistemas assistenciais próprios, prestando assistência médica e previdenciária de forma direta aos seus funcionários. O crescimento das Caixas de Assistências, nessa época, foi relevante, estendendo seus 
credenciamentos para atendimento cada vez maior a demandas externas às empresas. Esse crescimento gerou conflitos junto à categoria médica, provocando divisão de correntes de entendimento sobre a relação entre profissionais médicos e empresas prestadoras de assistência médica. De um lado, uma vertente defendia a prática liberal da medicina, de outro, a defesa da prática médica voltada ao mercado que se apresentava. Deu-se origem a duas modalidades de empresas médicas: as cooperativas médicas, que prestavam atendimento nos consultórios dos próprios profissionais, e as empresas de medicina de grupo, responsáveis pelos atendimentos hospitalares (Brasil, 2007).

Desde a década de 1960, trabalhadores de estatais, bancários, algumas instituições do governo federal, entre outros, já possuíam planos de saúde (Oliveira et al., 2007). A partir deste novo modelo já estabelecido no país, com características diversificadas, formatou-se a estrutura da saúde suplementar que viria a ser consolidada no país. Segundo Teixeira e colaboradores (2002), o processo de construção e consolidação das operadoras de saúde suplementar no Brasil evoluiu para a configuração, em linhas gerais, dos seguintes modelos:

1. Medicina de grupo: Gestão é feita por empresa privada, tendo os serviços prestados por unidades próprias ou credenciadas.

2. Empresas de seguro de saúde: São empresas que administram a relação entre beneficiários (pessoa física ou jurídica) e os prestadores de serviços de saúde. Não possuem rede própria e prestam os serviços de assistência de forma indireta, por meio da rede credenciada.

3. Cooperativa médica: São organizadas na forma de cooperativas pelos profissionais vinculados a entidades. Podem possuir hospitais próprios e têm como clientes pessoas físicas e jurídicas. Atualmente as cooperativas médicas estão organizadas em bases municipais, regionais e estaduais.

4. Empresas de autogestão: Nessas empresas em regra, os serviços estão voltados para seus empregados e familiares. Essa modalidade, também prevê a existência de empresas que constituem entidade sem fins lucrativos para prestação de serviços de assistência à saúde aos associados.

\subsection{A Saúde suplementar e a cobertura de fornecimento de medicamentos do componente especializado}

A Lei no 9.656/98 (Brasil, 2009), em seu art. 12, dispõe de forma objetiva a respeito da obrigatoriedade, por parte das operadoras de planos privados de saúde, sobre o fornecimento de medicamentos aos usuários dos planos que estiverem internados para tratamento Hospitalar:

Art. 12. São facultadas a oferta, a contratação e a vigência dos produtos de que tratam o inciso I e o $\S 1$ 을 do art. 1ํ desta Lei, nas segmentações previstas nos incisos I a IV deste artigo, respeitadas, as respectivas amplitudes de cobertura definidas no plano-referência de que trata o art. 10, segundo as seguintes exigências mínimas: 
(...)

I - quando incluir atendimento ambulatorial:

a) cobertura de consultas médicas, em número ilimitado, em clínicas básicas e especializadas, reconhecidas pelo Conselho Federal de Medicina;

b) cobertura de serviços de apoio diagnóstico, tratamentos e demais procedimentos ambulatoriais, solicitados pelo médico assistente;

II — quando incluir internação hospitalar:

\section{(...)}

d) cobertura de exames complementares indispensáveis para o controle da evolução da doença e elucidação diagnóstica, fornecimento de medicamentos, anestésicos, gases medicinais, transfusões e sessões de quimioterapia e radioterapia, conforme prescrição do médico assistente, realizadas ou ministradas durante o período de internação hospitalar.

Ainda a Lei no 9.656/98 (Brasil, 2009), em seu art. 10, excetua o fornecimento de medicamentos de uso domiciliar da obrigatoriedade de cobertura pelos planos privados de saúde. Nesse mesmo artigo, a lei também não prevê a realização de procedimentos como: fornecimento de medicamentos importados ou não nacionalizados, tratamento clínico ou cirúrgico experimental, tratamentos ilícitos ou antiéticos, assim definidos sob o aspecto médico, ou não reconhecidos pelas autoridades competentes. De acordo com Santos (2006), a regulação pública de saúde suplementar precisa continuar com o objetivo de torná-la autossuficiente e, portanto, com maiores possibilidades de respostas às ações integrais de assistência à saúde dos seus beneficiários. A ampliação das ações de saúde, notadamente aquelas que incidem significativamente na promoção da saúde e prevenção de agravos, deveria ser perseguida sistematicamente pelas operadoras dos planos privados de saúde visto que, comprovadamente, essas ações refletem maiores benefícios a todos (Bahia, 2001).

Nessas circunstâncias, onde existe, de um lado, a necessidade do uso de medicamentos importantes à prevenção, recuperação e tratamentos de agravos à saúde e, por outro, a legislação que isenta os planos privados de saúde da obrigação de fornecerem medicamentos para tratamento domiciliar, é evidente a necessidade de aprofundar as discussões e buscar soluções viáveis (Vianna et al., 2001).

\section{Procedimentos metodológicos}

\subsection{Tipo de estudo, população, período e fonte dos dados}

Trata-se de um estudo observacional e transversal sobre dados referentes às solicitações de medicamentos do componente especializado de residentes no estado de Minas Gerais, registrados no banco de dados da Secretaria Estadual de Saúde de Minas Gerais (SES/MG). Foram selecionados todos os processos de solicitação de medicamentos excepcionais deferidos no 
primeiro semestre de 2008 e que continham o número do Cadastro de Pessoa Física (CPF), bem como as variáveis descritas a seguir: nome, sexo, Gerência Regional de Saúde (GRS), nome do medicamento, dosagem, posologia, quantidade e diagnóstico com base na Classificação Estatística Internacional de Doenças (CID-10) (Di Nubila e Buchalla, 2008).

A existência do nome e número do CPF era condição imprescindível para o pareamento de dados entre os bancos da SES/MG e da Agência Nacional de Saúde Suplementar (ANS).

\subsection{Seleção da amostra}

O banco de dados dos solicitantes de medicamentos do componente especializado da SES/ MG foi construído a partir da base total de cadastro de usuários cujos processos de solicitação foram deferidos no primeiro semestre de 2008. Para esse período, constava nos registros da SES/MG o total de 15.358 solicitações de medicamentos.

Os dados inicialmente elegíveis para esse estudo, que incluíam obrigatoriamente nome e CPF, totalizaram 6.741 solicitações, correspondendo a 44\% de todas as solicitações do período. Esse arquivo foi gerado na forma de planilha Excel e foi enviado à Agência Nacional de Saúde Suplementar (ANS), onde foi utilizado o método Record Linkage com o banco geral de beneficiários cadastrados na referida Agência para verificar se os solicitantes eram ou não beneficiários de planos privados de saúde.

\subsection{0 pareamento dos dados da SES/MG e da ANS}

O método Record Linkage usado pela ANS é uma técnica de integração entre sistemas existentes, combinando dados de dois ou mais bancos para verificar a sobreposição desses dados, possibilitando, assim, a obtenção de um novo banco gerado como produto do cruzamento dos dados.

A ANS, por meio da sua Gerência de Produção de Informações, realizou o pareamento dos dados entre os dois bancos, conforme os passos a seguir:

Passo 1) Verificou-se inicialmente se os nomes dos solicitantes de medicamentos do componente especializado com seus respectivos CPFs (arquivo SES/MG) constavam do cadastro de CPFs da Receita Federal.

Passo 2) Foram removidos do arquivo SES/MG os registros inconsistentes por inexistência de $\mathrm{CPF}$ ou $\mathrm{CPF}$ divergente e com registro como usuários posteriores ao segundo semestre de 2008.

Passo 3) Foi realizado o cruzamento dos registros consistentes após o Passo 2 entre o banco SES/MG com os vínculos de usuários com cadastro no Sistema de Informações dos Beneficiários (SIB)/ANS, fazendo referência a informações sobre o primeiro semestre de 2008.

Passo 4) Para cumprimento dos requisitos éticos, todos os solicitantes de medicamentos que constavam como usuários de planos privados de saúde foram desidentificados por meio da remoção dos nomes e respectivos CPFs e receberam um código numérico. 
A amostra elegível para esse estudo ficou composta por 6.733 solicitações de medicamentos do componente especializado da assistência farmacêutica.

\subsection{Análise e tratamento dos dados}

A variável de origem da solicitação do medicamento teve como referência as Gerências Regionais de Saúde, totalizando 28 unidades administrativas localizadas nas cidades-polo do estado de Minas Gerais (Brasil, 2009).

Para conhecimento do perfil dos medicamentos mais frequentes nas solicitações foi utilizada a recomendação da Organização Mundial de Saúde (OMS) por meio da Anatomic Therapeutic Chemical Classification System, conhecido como Código ATC. Identificou-se o subgrupo farmacológico (nível 3 do Código ATC) e a substância química (nível 5 do Código ATC), relacionando o fármaco, a forma farmacêutica, a dosagem e a quantidade fornecida para um mês de tratamento. O Código ATC é adotado pela Organização Mundial de Saúde (OMS) por classificar os fármacos em diferentes grupos e subgrupos de acordo com o órgão ou sistema sobre o qual atua segundo suas propriedades químicas, farmacológicas e terapêuticas. Além de ser atualizado permanentemente a cada dois anos, o Código ATC tem compatibilidade internacional (Vidotti et al., 2008).

Para o estudo das patologias mais frequentes utilizaram-se os diagnósticos mencionados nas solicitações e agrupados em capítulos com base na CID-10. A relação entre os diagnósticos e os medicamentos está de acordo com os Protocolos Clínicos e Diretrizes Terapêuticas do Ministério da Saúde, utilizados para o deferimento das solicitações feitas à SES/MG (Brasil, 2009a).

Na quantificação dos gastos financeiros dos medicamentos fornecidos aos usuários de planos privados de saúde foi utilizada a tabela de preços dos medicamentos fornecidos pela SES/MG, no ano de 2008, constante no Banco de Preços em Saúde (BPS) do Ministério da Saúde. Essa tabela relaciona os valores definidos pela menor unidade posológica, adquiridos pela SES/MG no ano de 2008. O valor por medicamento fornecido é o produto do valor unitário pela quantidade dispensada.

Num primeiro momento, as informações foram analisadas por meio de técnicas de estatística descritiva, com o objetivo de organizar os dados. Foram construídas tabelas simples, de distribuição de frequência e de contingência, gráficos, histograma e diagrama de Pareto.

Foram apresentados cálculos de porcentagens das categorias para as variáveis envolvidas no estudo. Para o resumo dos dados sobre custos com os medicamentos, foram calculados valores para média, mediana, menor custo, maior custo, desvio-padrão e o respectivo coeficiente de variação. Valores de percentis (p5, p10, p90 e p95) também foram obtidos, com intuito de serem apresentados possíveis valores de referência para o custo. As comparações entre os tipos de solicitações em relação ao custo com medicamentos foram feitas por meio de teste de hipótese não paramétrico (Mann-Whitney), considerando nível de significância de $5 \%(\alpha=0,05)$. A análise multivariada foi feita por meio de regressão linear múltipla (Altman, 1991). 


\subsection{Aspectos éticos da pesquisa}

Este projeto de pesquisa foi submetido e aprovado pelo Comitê de Ética em Pesquisa da Universidade Federal de Minas Gerais (Coep-UFMG) sob o parecer no Caae 046/2010, com dispensa de aplicação do Termo de Consentimento Livre e Esclarecido (TCLE). Ficaram garantidos o sigilo da identificação das pessoas envolvidas nos processos de solicitações de medicamentos e a total confidencialidade das informações.

\section{Resultados e discussão}

Neste estudo foram analisadas 6.733 solicitações de medicamentos excepcionais à SES/MG, feitas por 6.076 usuários entre janeiro e junho de 2008. Dessas, 2.058 (31\%) foram feitas por usuários de planos privados de saúde. O custo com os usuários com planos privados de saúde representou $37 \%$ de todo o gasto com os medicamentos solicitados, conforme mostrado no gráfico 1.

\section{Gráfico 1}

Custo das solicitações de medicamentos excepcionais por usuários com e sem planos privados de saúde.

Minas Gerais, janeiro a junho de 2008

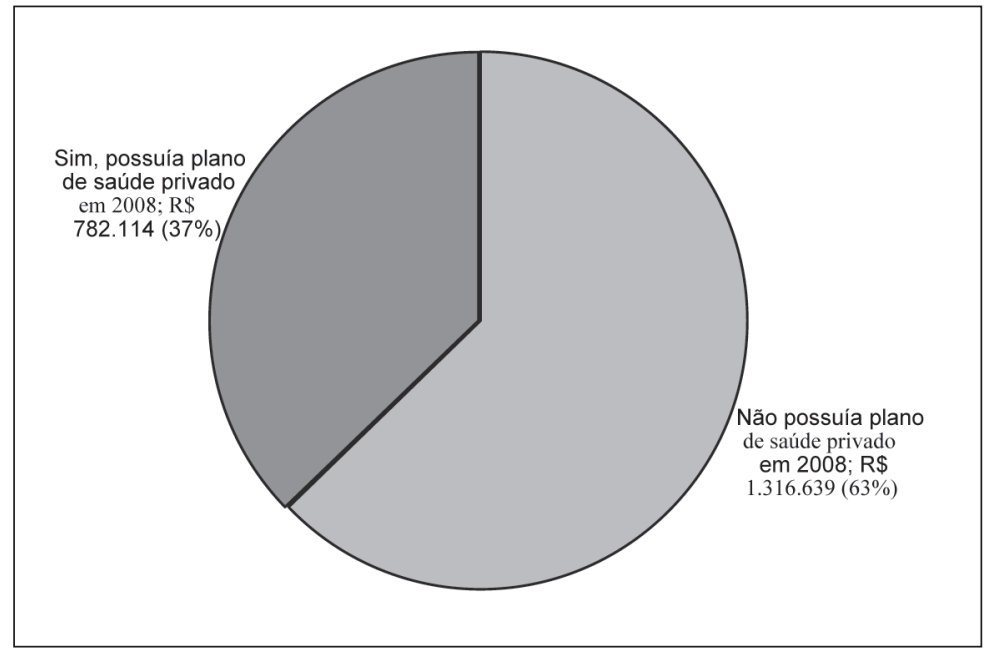

Fonte: Elaborado pelos autores com base nos dados do estudo.

Quanto à origem das solicitações (área de residência), tendo como referência as 28 Gerências Regionais de Saúde (GRS), o estudo demonstrou que a GRS Belo Horizonte, que abrange a Região Metropolitana, apresentou custo mais elevado quando comparado às so- 
licitações originadas nas demais GRS do interior do estado, com diferença estatisticamente significativa $(\mathrm{p}<0,01)$. A GRS de Belo Horizonte apresentou ainda maior percentual de solicitações na faixa de custos acima de $\mathrm{R} \$ 1.500,00$ e menor percentual abaixo de $\mathrm{R} \$ 100,00$ como pode ser verificado no gráfico 2 .

\section{Gráfico 2}

Distribuição percentual das solicitações de medicamentos excepcionais por faixa de custo nas GRS BH e demais GRS do interior do Estado. Minas Gerais, janeiro a junho de 2008

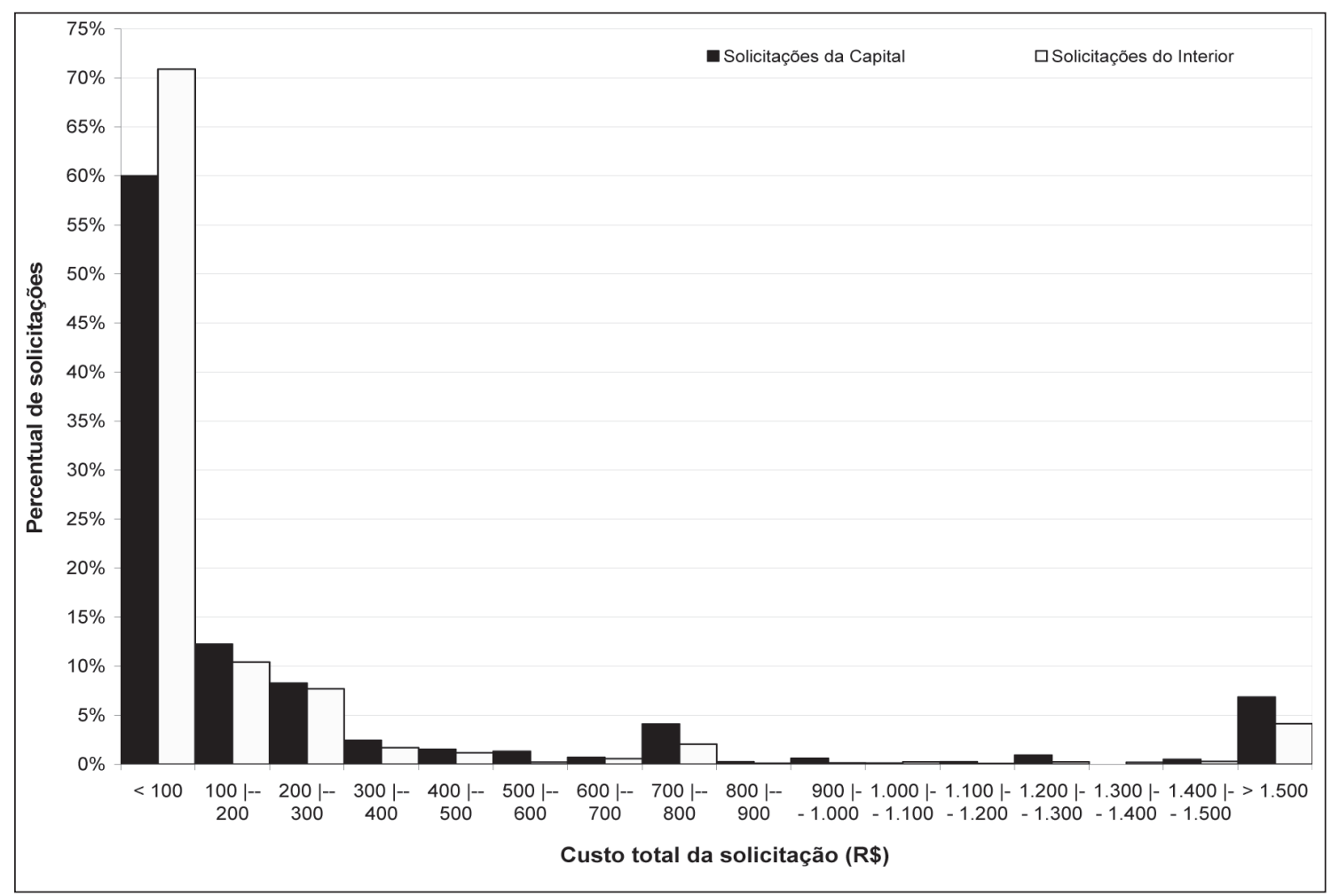

Fonte: Elaborado pelos autores com base nos dados do estudo.

Constatou-se também, como mostra a tabela 1, que o custo mediano dos medicamentos excepcionais solicitados por usuários de planos foi mais elevado $(\mathrm{R} \$ 61,20)$ em relação ao custo das solicitações de quem não tinha plano ( $\mathrm{R} \$ 48,00)$, com diferença estatisticamente significativa $(\mathrm{p}<0,01)$. Verifica-se ainda na tabela 1 diferença significativa apontando que $80 \%$ dos medicamentos fornecidos aos beneficiários de planos privados estão entre os valores de $\mathrm{R} \$ 10,00$ e R\$782,00, enquanto para os não beneficiários de planos esse mesmo percentual (80\%) está entre os valores de R\$ 6,00 e R\$ 469,00. 
Tabela 1

Custo das solicitações de medicamentos excepcionais feitas por beneficiários com e sem planos privados de saúde. Minas Gerais, janeiro a junho de 2008

\begin{tabular}{|ccc|}
\hline SOLICITAÇÕES & COM PLANO & SEM PLANO \\
\hline Total de solicitações analisadas $=$ & 2.058 & 4.675 \\
Solicitação com menor custo $=$ & 0,05 & 0,20 \\
Solicitação com maior custo $=$ & $24.186,00$ & 15201,00 \\
Custo médio $=$ & 380,03 & 281,63 \\
Custo mediano $=$ & 61,20 & 48,00 \\
Desvio-padrão $=$ & $1.141,58$ & 886,70 \\
Coeficiente de variação $=$ & $300 \%$ & $315 \%$ \\
Percentil $5=$ & $R \$ 2$ & $R \$ 2$ \\
Percentil $10=$ & $R \$ 10$ & $R \$ 6$ \\
Percentil $90=$ & $R \$ 782$ & $R \$ 469$ \\
Percentil $95=$ & $R \$ 3.213$ & $R \$ 1.313$ \\
\hline
\end{tabular}

Fonte: Elaborada pelos autores com base nos dados do estudo.

A análise multivariada mostrou com diferença significativa $(\mathrm{p}<0,01)$ por regressão linear múltipla que as variáveis associadas ao custo dos medicamentos fornecidos pela SES/MG foram a origem da solicitação e ser usuário de plano privado de saúde, conforme evidenciado na tabela 2.

Tabela 2

Fatores associados ao custo das solicitações de medicamentos excepcionais fornecidos pela SES. Minas Gerais, janeiro a junho de 2008

\begin{tabular}{|lccc|}
\hline \multicolumn{1}{|c}{ Variável explicativa } & Coeficiente de regressão & Erro padrão do coeficiente de regressão & Valor-p \\
\hline Sexo do paciente & $+0,02$ & 0,043 & 0,662 \\
Origem da solicitação & $+0,38$ & 0,045 & $<0,001$ \\
Plano privado de saúde & $+0,38$ & 0,111 & $<0,001$ \\
\hline
\end{tabular}

Fonte: Elaborada pelos autores com base nos dados do estudo.

Nota: variável resposta: logaritmo neperiano do custo $=\ln$ (custo)

a análise de resíduo não apresentou qualquer anomalia

O estudo mostrou que parcela importante das solicitações de medicamentos do componente especializado à rede pública (SES/MG) foi feita por pacientes, usuários de planos privados de saúde. O percentual (31\%) de solicitantes cadastrados em planos privados de saúde em Minas Gerais foi superior ao geral registrado na população brasileira. Registros do IBGE apontam que 48,66 milhões de pessoas no Brasil utilizam planos privados, correspondendo a 25,9\% da população (Brasil, 2013b). 
Tendo como base a origem das solicitações de medicamentos por Gerências Regionais de Saúde, ficou evidente que a demanda por medicamentos do componente especializado é uma característica comum em todo o estado de Minas Gerais, confirmando os achados de Dantas e Silva (2006). Todavia, ficou demonstrada a grande proporção de solicitações originadas pela Gerência Regional de Saúde de Belo Horizonte, cujo número aproxima-se da soma de todas as solicitações originadas nas demais gerências do estado. Algumas justificativas para a maior demanda por medicamentos nas regiões metropolitanas seriam a maior divulgação e acesso às informações, tanto por parte dos pacientes quanto dos médicos prescritores, sobretudo, quanto à informação da não exigência por parte da SES/MG de que a prescrição do medicamento e o preenchimento de formulários tenham que ser realizados somente por médicos vinculados ao SUS. Verificou-se ainda que as solicitações originadas na Região Metropolitana da capital mineira apresentaram maior frequência para os medicamentos mais caros em comparação com as demais gerências regionais do estado, reforçando os resultados de Dantas e Silva (2006) sobre o acesso às informações dos medicamentos disponibilizados pelo sistema público.

Foi expressiva a parcela de recursos financeiros dispendida ao fornecimento de medicamentos do componente especializado aos beneficiários de planos privados de saúde. Os dados mostram que $37,2 \%$ dos valores gastos dizem respeito às solicitações feitas por beneficiários com plano privado (31\%). Verifica-se também que os custos medianos de medicamentos solicitados por beneficiários foram mais elevados que aqueles solicitados por não beneficiários de planos. Resultados similares foram encontrados por Albuquerque (2008) ao discutir a relação econômica dos beneficiários de planos de saúde e a busca pelos serviços públicos de saúde.

Ao demonstrar que as solicitações dos beneficiários com planos apresentaram menor frequência para medicamentos mais baratos (valores abaixo de R\$100,00) e maior frequência para medicamentos mais caros (valores acima de $R \$ 1.500,00$ ) quando comparadas às solicitações de não beneficiários, o estudo alerta para a necessidade de identificação das reais causas geradoras dessas diferenças de custos.

Observou-se também a grande variabilidade entre as solicitações de medicamentos do componente especializado com maior e menor preço. O estudo deixa evidente (tabela 1) que os custos dos medicamentos fornecidos para beneficiários ou não de planos privados apresentam significativa diferença quando comparados por faixa de percentis, onde $80 \%$ das solicitações dos beneficiários de planos estavam com custos na faixa entre $R \$ 10,00$ e $R \$ 782,00$ ao passo que $80 \%$ dos custos das solicitações dos não beneficiários estavam entre R \$ 6,00 e R\$ 469,00. A análise desses percentis pode contribuir de maneira importante ao melhor planejamento da gestão econômica sobre os medicamentos fornecidos pela rede pública de saúde.

Os custos médio e mediano das solicitações também foram mais elevados para os beneficiários de planos de saúde, com diferença estatisticamente significativa $(\mathrm{p}<0,01)$ quando comparados aos beneficiários sem plano. É importante observar que o custo mediano representa de forma mais fidedigna os gastos com o fornecimento de medicamentos em função da distribuição assimétrica do custo, conforme visto no estudo. Esses achados estão em consonância com estudos feitos por Dias e Romano-Lieber (2006) e Oliveira e colaboradores (2007) 
sobre acesso a medicamentos no Brasil. Esses estudos demonstram a preferência de grupos da população pertencente às classes com maior poder aquisitivo (classes A e B), beneficiários de planos privados de saúde, por medicamentos com preços mais elevados (com nomes de marcas tradicionais) em relação aos medicamentos genéricos.

Como demonstrado por Malta e colaboradores (2004), recomenda-se que, para evitar a fragmentação do cuidado, se torne necessário que os prestadores de serviços de saúde, públicos ou privados, devam operar em outro formato do modelo assistencial, comandado por um processo de trabalho cuidador, que oriente esse percurso. Quando isso não existe, o usuário faz o seu próprio caminhar pelas redes de serviços, induzindo consumo de procedimentos. O grande desafio consiste em restabelecer uma nova prática, centrada no estímulo à promoção da saúde, prevenção, referenciada no vínculo e na responsabilização.

\section{Considerações finais}

Os dados apontam para a necessidade de discussão e implementação da gestão da política de assistência farmacêutica pelos planos privados de saúde que minimizem os gastos públicos nesse setor e viabilizem o acesso dos beneficiários de planos privados aos medicamentos. Essa perspectiva vem ao encontro da defesa de um modelo de assistência à saúde que estabeleça ações preventivas, incluindo a utilização racional de medicamentos como prevenção aos agravos à saúde.

A atual situação em que se encontra a prática de assistência farmacêutica desenvolvida pelos planos privados de saúde configura-se em importante e urgente desafio a ser debatido e superado pelos diversos atores envolvidos, com destaque para os usuários dos serviços de saúde, organismos de defesa do consumidor e do controle social, o Poder Legislativo, gestores públicos e certamente pelos administradores das operadoras de planos privados no Brasil.

Por fim, o estudo possibilitou concluir que a demanda por medicamentos do componente especializado pelos beneficiários de planos privados de saúde junto ao serviço público é real e que os custos são mais elevados quando comparados às solicitações feitas por beneficiários sem planos privados. Concluiu-se também que solicitações desses medicamentos originadas na Região Metropolitana de Belo Horizonte apresentaram maior frequência e custos mais elevados em relação às demais regiões do estado de Minas Gerais.

\section{Referências}

ALBUQUERQUE, Ceres et al. A situação atual da saúde suplementar no Brasil e apontamentos para o futuro. Ciência \& Saúde Coletiva, Rio de Janeiro, v. 13, n. 5, p. 1421-1430, set./out. 2008.

ALTMAN, Douglas G. Practical statistics for medical research. Londres: Chapman \& Hall CRC, 1991. 
ANGELL, Marcia. A verdade sobre os laboratórios farmacêuticos. Tradução de Waldéa Barcellos. 3. ed. Rio de Janeiro: Record, 2008.

AQUINO, Daniela S. Por que o uso racional de medicamentos deve ser uma prioridade? Ciência \& Saúde Coletiva, Rio de Janeiro, v. 13, n. Sup., p. 733-736, 2008.

BAHIA, Lígia. Planos privados de saúde: luzes e sombras no debate setorial dos anos 90. Ciências \& Saúde Coletiva, v. 6, n. 2, p. 329-339, 2001.

BARROS, José A. C. Políticas farmacêuticas: a serviço dos interesses da saúde? Brasília: Organização das Nações Unidas para a Educação, a Ciência e a Cultura, 2004.

BRASIL. Agência Nacional de Saúde Suplementar. Caderno de informação da Saúde Suplementar. Rio de Janeiro: ANS, 2013a.

BRASIL. Conferência Nacional de Medicamentos e Assistência Farmacêutica. Relatório final: efetivando o acesso, a qualidade e a humanização da assistência farmacêutica, com controle social. Brasília: Ministério da Saúde, Conselho Nacional de Saúde, 2005.

BRASIL. Constituição da República Federativa do Brasil. 2. ed. Rio de Janeiro: América Jurídica, 2006.

BRASIL. Conselho Nacional de Secretários de Saúde. Assistência farmacêutica no SUS. Brasília: Conass, 2007. (Progestores — Para entender a gestão do SUS, 2007).

BRASIL. Conselho Nacional de Secretários de Saúde. Saúde Suplementar/Conselho Nacional de Secretários de Saúde. Brasília: Conass, 2007. (Progestores — Para entender a gestão do SUS, 2007).

BRASIL. Lei n. 8.080 de 11 de dezembro de 2000. Dispõe sobre a regulamentação do Sistema Único de Saúde e dá outras providências. Diário Oficial da União, Brasília, 12 dez. 2000.

BRASIL. Lei n. 9.656 de 03 de junho de 1998. Dispõe sobre os planos e seguros privados de assistência à saúde. Disponível em: <www.planalto.gov.br/CCIVIL/leis/L9656.htm>. Acesso em: 16 jul. 2009.

BRASIL. Ministério da Saúde. Diretrizes Operacionais dos Pactos pela vida, em defesa do SUS e de gestão. Ministério da Saúde. Secretaria Executiva, Departamento de Apoio à Descentralização. Coordenação-Geral de Apoio à Gestão Descentralizada. Brasília, 2006.

BRASIL. Ministério da Saúde. Gabinete do Ministro. Portaria n 2.981, de 26 de novembro de 2009. Aprova o Componente Especializado da Assistência Farmacêutica. Brasília, 2009a.

BRASIL. Ministério da Saúde. Protocolos clínicos e diretrizes terapêuticas: medicamentos excepcionais. Ministério da Saúde. Secretaria de Assistência à Saúde, Departamento de Sistemas e Redes Assistenciais. Brasília: Ministério da Saúde, 2002.

BRASIL. Ministério da Saúde. Secretaria de Vigilância em Saúde. Secretaria de Gestão Estratégica e Participativa. Vigitel Brasil 2009: vigilância de fatores de risco e proteção para doenças crônicas por inquérito telefônico. Brasília: Ministério da Saúde, 2010. (Série G. Estatística e Informação em Saúde). 
BRASIL. Ministério do Planejamento, Orçamento e Gestão. Plano mais Brasil PPA 2012-2015: relatório anual da avaliação: ano base 2012/Ministério do Planejamento, Orçamento e Gestão/SPI. Brasília: MP/SPI, 2013b.

BRASIL. Secretaria de Assistência à Saúde, Minas Gerais. Protocolos clínicos e diretrizes terapêuticas: medicamentos excepcionais. Disponível em: <www.opas.org.br/medicamentos/docs/pcdt/05_protocolos.pdf $>$. Acesso em: 7 mar. 2011a.

BRASIL. Secretaria Estadual de Saúde, Minas Gerais. Construindo ações de saúde do trabalhador no âmbito das superintendências e gerências regionais de saúde. Disponível em: <www.nescon. medicina.ufmg.br/biblioteca/imagem/2839.pdf>. Acesso em: 7 mar. 2011b.

CARVALHO, Maria Cleide R. D.; JÚNIOR, Horácio A.; RAFFIN, Fernanda N. Representações sociais do medicamento genérico por consumidores em Natal, Rio Grande do Norte. Cad. Saúde Pública, Rio de Janeiro, v. 22, n. 3, p. 653-661, 2006.

DANTAS, Nara S.; SILVA, Ramiro R. Medicamentos excepcionais. Brasília: Escola Superior do Ministério Público da União, 2006.

DI NUBILA, Heloisa B. V.; BUCHALLA, Cassia Maria. O papel das Classificações da OMS — CID e CIF nas definições de deficiência e incapacidade. Rev. Bras. Epidemiol., São Paulo, v. 11, n. 2, p. 324-335, jun. 2008.

DIAS, Cláudia Regina C.; ROMANO-LIEBER, Nicolina Silvana. Processo da implantação da política de medicamentos genéricos no Brasil. Cad. Saúde Pública, Rio de Janeiro, v. 22, n. 8, p. 1661-1669, 2006.

FIBGE. Fundação Instituto Brasileiro de Geografia e Estatística. Pesquisa Nacional por Amostra de Domicílios: um panorama da saúde no Brasil. Acesso e utilização dos serviços, condições de saúde e fatores de risco e proteção à saúde ano 2008. Rio de Janeiro: Fiocruz, 2010.

LEFRÉVE, Fernando; LEFRÉVE, Ana Maria C. Saúde, empoderamento e triangulação. Saúde e Sociedade, São Paulo, v. 13, n. 2, p. 32-38, maio/ago. 2004.

MALTA, Deborah C. et al. Perspectivas da regulação na saúde suplementar diante dos modelos assistenciais. Ciências \& Saúde Coletiva, Rio de Janeiro, v. 9, n. 2, p. 433-444, abr./jun. 2004.

MARTINS, André. Biopolítica: o poder médico e a autonomia do paciente em uma nova concepção de saúde. Interface - Comunicação, Saúde, Educação, Botucatu, v. 8, n. 14, p. 21- 32, set. 2003/ fev. 2004.

MONTONE, Januário. Coletânea de artigos e palestras sobre o setor da Saúde Suplementar no Brasil, 2000 a 2003. Rio de Janeiro: Ministério da Saúde/Agência Nacional de Saúde Suplementar, 2004.

NASCIMENTO, Marilene C. A centralização do medicamento na terapêutica contemporânea. Tese de (doutorado em saúde coletiva) - Instituto de Medicina Social, Universidade do Estado do Rio de Janeiro, Rio de Janeiro, 2002.

OLIVEIRA, Maria A. et al. Assistência farmacêutica e acesso a medicamentos. Rio de Janeiro: Fiocruz, 2007. 
PERINI, Edson. Assistência farmacêutica: fundamentos teóricos e conceituais. In: ACURCIO, Francisco A. (Org.). Medicamentos e assistência farmacêutica. Belo Horizonte: Coopmed, 2003. p. 31-60.

RODRIGUES, Joelson T. A medicação como única resposta: uma miragem do contemporâneo. Psicologia em Estudo, Maringá, v. 8, n. 1, p. 13-22, jan./jun. 2003.

SANTOS, Fausto P. A regulação pública da saúde no Brasil: o caso da saúde suplementar. Tese (doutorado) - Programa de Pós-Graduação em Saúde Pública, Faculdade de Ciências Médicas, Universidade Estadual de Campinas, Campinas, 2006.

SOARES, Jussara C. R. S. Quando o anúncio é bom, todo mundo compra. O Projeto MonitorAÇÃO e a propaganda de medicamentos no Brasil. Ciência \& Saúde Coletiva, Rio de Janeiro, v. 13, n. sup., p. 641-649, 2008.

TEIXEIRA, Aloísio et al. Nota sobre a regulação dos planos de saúde de empresas, no Brasil. In: REGULAÇÃO \& Saúde: estrutura, evolução e perspectivas da assistência médica suplementar. Ministério da Saúde, Agência Nacional de Saúde Suplementar. Rio de Janeiro: ANS, 2002. p. 19-35.

VIANNA, Cid M. M. et al. Atenção básica a saúde: consolidação do modelo de fornecimento de medicamentos pelo segmento de operadoras de planos de saúde: um cenário do Benefício Farmacêutico no Brasil. Relatório parcial, ANS, 2001.

VIDOTTI, Carlos C. F. et al. New drugs in Brazil: do they meet Brazilian public health needs? Revista Panamericana de Salud Publica, Washington D.C., v. 24, n. 1, p. 36-45, 2008.

Rilke Novato Públio é mestre em enfermagem pela Universidade Federal de Minas Gerais (UFMG). Email:rnpublio@yahoo.com.br.

Braulio Roberto Gonçalves Marinho Couto é doutor em bioinformática pela Universidade Federal de Minas Gerais (UFMG) e professor do Centro Universitário Belo Horizonte (UNI-BH). E-mail: coutobraulio@gmail.com.

Analina Furtado Valadão é doutora em bioquímica e imunologia pela Universidade Federal de Minas Gerais (UFMG) e professora do Instituto Metropolitano do Ensino Superior (Imes/Univaço). E-mail: analina@famevaco.br.

Edna Maria Rezende é doutora na área de epidemiologia e professora associada da Escola de Enfermagem da Universidade Federal de Minas Gerais (UFMG). E-mail: ednarez@ufmg.br. 